\title{
Regional orthogonal models of the geomagnetic field changes over the Far East
}

\author{
Irina A. Burdelnaya ${ }^{1}$, Sergey V. Filippov ${ }^{1}$, Vadim P. Golovkov ${ }^{1}$, Satoshi Fujiwara ${ }^{2}$, Tadashi Tanabe ${ }^{2}$, \\ Shujiro Nishi ${ }^{2}$, Masaru Kaidzu², and Shigeru Matsuzaka² \\ ${ }^{1}$ Institute of Terrestrial Magnetism, Ionosphere and Radiowave Propagation, Russian Academy of Sciences (IZMIRAN), \\ Troitsk, Moscow region 142092, Russia \\ ${ }^{2}$ Geographical Survey Institute, Kitasato 1, Tsukuba, Ibaraki 305-0811, Japan
}

(Received May 18, 1998; Revised December 14, 1998; Accepted March 2, 1999)

\begin{abstract}
Two spatial-temporal models of the geomagnetic field secular variations in a time interval from 1970.0 to 1995.0 were constructed for the Far East region using the technique of regional modeling. First model covers a rectangular area, which includes Kamchatka, Sakhalin, the Kuril and Japanese Islands, the Ohotsk and the Japan Seas. Data from Russian, Japanese, and partially, Chinese observatories, and repeat stations were used for its construction. For filling of no data areas (the seas) the global models were used. Second model covers the area of the Japanese Islands and, partially, surrounding seas. Data from the Japanese magnetic observatories and first class repeat station network were utilized for its construction. We used natural orthogonal components method for obtaining temporal and spatial functions of the models. The accuracy of obtained models is much better than that of global models.
\end{abstract}

\section{Introduction}

For last years the requirements for high accuracy of mathematical modeling of the geomagnetic field and its secular variations (SV) were increased considerably. It is connected with requests from fundamental research fields, such as practice of magnetic anomaly mapping. Indeed the researches on high-frequency secular variations due to internal origin and on feature of geomagnetic jerks are in need of spatialtemporal models with accuracy a few nT. The same accuracy is necessary for reducing of data of large-scale magnetic surveys from epoch of survey to one of mapping. But an accuracy of temporal models as global, and/or as regional is not better of many tens nT up today (Haines, 1990).

The accuracy of models depends first of all on quantity and accuracy of initial data. On huge ocean areas a net of repeat stations and magnetic observatories is very rare and therefore the accuracy of models over ocean regions can not be high. The global models, such as international geomagnetic reference fields (IGRF), give smoothed value of accuracy on all surface of the Earth.

Only for some large regions, such as Northern America, Europe, Australia and separate regions of Asia, where we have the data amount being satisfactory, the spatial-temporary models of enough high accuracy can be made. Present study is an attempt to create such model for the Far East region using the data from the magnetic observatories as well as data from repeat stations, located inside a region of modeling.

\section{The Basic Statements}

Spatial-temporal modeling of changes in the geomagnetic

Copy right (c) The Society of Geomagnetism and Earth, Planetary and Space Sciences (SGEPSS); The Seismological Society of Japan; The Volcanological Society of Japan; The Geodetic Society of Japan; The Japanese Society for Planetary Sciences. field on the Earth's surface can be described as follows:

$$
H(\varphi, \lambda, t)=\sum_{i=0}^{I} \sum_{j=0}^{J} \sum_{k=0}^{K} a_{i j k} F(\varphi)_{i} \cdot G_{j}(\lambda) \cdot D_{k}(t) .
$$

Where $\varphi$ and $\lambda$ are the geographical coordinates, $t$ is the time (years or other units); $F, G$ and $D$ are some functions being usually analytical ones.

The task of choosing what kind of functions is main at modeling. We consider the common requirements at choosing the functions for spatial descriptions of the geomagnetic field in a limited area. It is known that the observed field is a sum of fields due to different origin:

$$
H=H_{\mathrm{n}}+H_{\mathrm{a}}+\delta_{\mathrm{n}} .
$$

Where $H_{\mathrm{n}}$ is the normal fields, $H_{\mathrm{a}}$ is the anomalous field and $\delta_{\mathrm{n}}$ is errors in the observations. The distinctions of the normal components, $H_{\mathrm{n}}$, and the anomalous ones, $H_{\mathrm{a}}$, consist not only in their natures, but also in the frequency structure. From the point of view of the mathematical statistics, the measured field is a sum of high-frequency part being determined as the magnetic anomalies, and the low-frequency part representing the normal field. The analytical functions of the model describe usually the low-frequency part and the procedure of their obtaining is thus a sort of the frequency filter. At appropriate choosing of the frequency characteristics such division can reflect the real division of the field on parts of different origin — on the field, generated in the Earth's core, and the field, connected with magnetized rocks of the Earth crust. Thus, choosing of analytical functions for modeling is based on real properties of the field and reflects the natural requirement of researchers.

But the aim of the temporal modeling is quite different. Except of local changes of the geomagnetic field, connected with tectonomagnetic phenomenon, magnetic anomalies do not change in time. All the temporal changes are stipulated 
by two kinds of sources. One of them is a process in the liquid core of the Earth. And the second is a process in the ionosphere and magnetosphere activated by the solar activity. These types of sources generate the magnetic fields, which have different frequency structures and amplitudes, but lie in identical range of space sizes. The space sizes of inner fields lie in limits from all surface of the Earth to 60-80 angular degrees. Their characteristic times are from units to millions of years, and their amplitudes of changes reach 100 and more nT per year.

Sources of the external origin generate fields of the same space characteristic. Their ranges of periods are 11 years and shorter and their amplitudes are 10-20 nT per year. Thus, only the normal field changes. Hence, to calculate the model, which describes the temporal changes with sufficient accuracy, we should refuse the smoothing and any other frequency filtering of data. The temporal functions in Eq. (1) should describe the changes in the geomagnetic field year by year with accuracy not worse than several nanoteslas. Obviously, any analytical functions do not satisfy this condition if the quantity of terms of exception (so, and quantity of parameters of the model) lies in reasonable limits. Nevertheless magnetologists use analytical functions for description of the secular variation, but we should emphasize that it is rather a forced choice. This contradiction can be permitted only if function $D(t)$ in Eq. (1) will be numerical ones.

The other requirement for the spatial-temporal modeling arises in connection with necessity to use methods of the mathematical statistics for obtaining parameters of a model. Usually, the method of least square is used. Equation (1) is conditional one. The left part in this equation, the observed value at a point with coordinates, $\varphi$ and $\lambda$ and the right part is the sum, in which functions $F, G$ and $D$ are defined at this point and coefficients $a_{i j k}$ are assumed as the unknowns. Let us rewrite (1) in the form:

$$
H(\varphi, \lambda, t)=\sum_{m=1}^{M} b_{m} E_{m}(\varphi, \lambda, t) .
$$

Where $b_{m}=a_{i j k}, E_{m}(\varphi, \lambda, t)=F_{i}(\varphi) G_{j}(\lambda) D_{k}(t)$, and $m$ is number of some combination from $i, j$ and $k$. In the method of least squares, the system of linear equations (1) is transformed to the system of normal equations, whose elements are presented below:

$$
\left|\mathbf{A}_{l m}\right|=\sum E_{l}(\varphi, \lambda, t) \cdot E_{m}(\varphi, \lambda, t) .
$$

Where $l$ is the number of a column and $m$ is the number of a row, $l$ and $m \in[1, M]$. It is clear that the matrix $\left|\mathbf{A}_{l m}\right|$ is reduced to diagonal one if functions $E_{m}$ in the given area are orthogonal, i.e. if the following condition fulfills:

$$
\int E_{m}(x) \cdot E_{l}(x) d x \begin{cases}=0, & l \neq m, \\ \neq 0, & l=m .\end{cases}
$$

Certainly, the transition from integral to summation makes the orthogonal condition of approximate. In this case all nondiagonal elements of matrix $\left|\mathbf{A}_{l m}\right|$ can be expected for being much less than diagonal ones. This simplifies not only the accounting task, but also makes the solution less dependent on quantity of members of the exception, as well as from quantity and the particular spatial distribution of data etc. It means that the requirement orthogonality for functions $F, G$ and $D$ on all area of modeling is very important.

One more requirement for functions choosing follows from rules of statistics. A quantity of the determined model parameters must be much less than that of initial data. This requirement limits the number of terms of the expansion, and consequently, it limits, for example, opportunity of analytical functions to fulfill the condition of the optimum frequency filter for division of the field on the normal and the anomalous parts. The last requirement, in the meaning of practical use, is to simplify functions, and consequently their computation.

\section{Choosing of a Basis of Temporal Functions}

As it was mentioned in the previous section, to avoid loss of accuracy due to smoothing of temporal variations of the geomagnetic field, they are necessary to be simulated by numerical functions. These functions must make the orthogonal basis. The natural orthogonal components (NOC) satisfy this condition (Fainberg, 1975). A method of such functions obtaining is stated below. The orthogonal function NOC's are different from various methods of decomposition and use the basic system of functions, which is built on the basis of the statistical structure of a studied object. In common case the experimental data make a two-dimensional table. In our case the data matrix has spatial-temporal character and presents the set of $J$ temporal series of the duration of $I$ numbers in each. Thus, the matrix is rectangular one of $I \times J$ elements, where $I$ is the number of column and $J$ is the number of row. The observed value of the field is recorded as follows:

$$
H_{i j}=\sum_{k} X_{k j} T_{k i}+\delta_{i j}
$$

Where $j$ is the number of a row and $i$ is the number of a column.

The algorithm of NOC calculation results from the proof of the orthogonality of the temporal functions $T_{k i}$ at the condition of the orthogonality of $X_{k j}$ :

$$
\begin{gathered}
\text { From } \begin{array}{c}
\sum_{i} X_{k i} X_{l i} \equiv 0 \quad \text { if } k \neq l \\
\sum_{i} X_{k i} X_{l i} \neq 0 \quad \text { if } k=l, \\
\text { it follows } \sum_{j} T_{k j} T_{l j} \equiv 0 \quad \text { if } k \neq l \\
\sum_{j} T_{k j} T_{l j} \neq 0 \quad \text { if } k=l .
\end{array}
\end{gathered}
$$

The proof of this condition is described in Fainberg (1975).

It is following from supposition that there are $N$ curves given by the vectors (Vertlib and Vagner, 1970; Golovkov et al., 1992):

$$
H^{(1)}=\left(H_{1}^{(1)}, \ldots, H_{M}^{(1)}\right), \ldots, H^{(N)}=\left(H_{1}^{N}, \ldots, H_{M}^{(N)}\right)
$$

which represent the time series of the geomagnetic field $\left(t_{1}, \ldots, t_{M}\right)$ at different observatories with coordinates $X_{1}, \ldots, X_{N}$. We should find such an optimum system of the orthogonal functions which provide the maximum rate of convergence for the given expansion:

$$
H_{i j}(t, x)=\sum_{k} T_{k i} X_{k j}, \quad(k<N) .
$$


Here $T_{k i}$ are the time functions and $X_{k j}$ are the natural functions which depends on the coordinates $\left(X_{1}, \ldots, X_{N}\right)$ and form the orthogonal system. Assuming that the rootmean-square error in approximation of the $H$ function should be minimum:

$$
\sum_{i} \sum_{j}\left[H_{i j}-\sum_{k} T_{k i} X_{k j}\right]^{2}=\min
$$

and equating their partial derivatives to zero, we obtain:

$$
T_{k i}=\frac{\sum_{j} H_{i j} X_{k j}}{\sum_{j} X_{k j}^{2}}
$$

and

$$
\begin{gathered}
\sum_{j} A_{k j} X_{l j}=\lambda_{l} X_{k l}, \\
A_{i k}=\sum_{j} H_{i j} H_{i k} \quad \text { and } \quad \lambda_{l}=\sum_{i} T_{l i}^{2} \sum_{j} X_{l j}^{2} .
\end{gathered}
$$

Equation (11) has a solution with $N$ eigenvalues $\lambda_{l}$ and corresponding eigenfunctions $X_{k l}$. From general matrix theory it follows that the $X_{k l}$ forms the orthogonal system (6) and, according to (12), $\lambda_{l}$ is a number of decreasing dispersions of the expansion coefficients on the eigenfunctions.

From (6) and (10) we deduce the orthogonality of the time functions:

$$
\sum_{i} T_{l i} T_{g i}=0 \quad \text { for } \quad l \neq g
$$

Thus, all eigenfunctions and all time functions are linearly independent. Therefore, different members of the expansion (8) describe fields of different sources.

The described functions $X_{k j}$ and $T_{k j}$ do not depend on rearrangement of rows or columns of the original table of data. Obviously, $X_{k j}$ in Eq. (8) are not any functions of coordinates, but only functions of a point of the observation. It is possible to think, that in each point of observation supervision $i$ and $j$ the measured value with some error minimized on all points is simulated by linear combination of some temporal functions $T_{k i}$, where $X_{k i}$ fulfills a role of a coefficient. We emphasize once again that the condition of the orthogonality $T_{k i}$ is fulfilled on given set. Also obviously, that the abovestated expansion is not a frequency filter and each temporal function can contain whole spectrum of frequencies.

Errors $\delta_{i j}$ are allocated not on frequency principle, but on attribute of their non covariance in temporal series. It is important also to emphasize that the different temporal functions can contain the same frequency, if the condition of the orthogonality is fulfilled. For example, the functions sin and cos, as far as can be divided:

$$
\int_{t_{1}}^{t_{2}} \sin \omega t \cos \omega t d t=0
$$

Hence, NOC's completely satisfy the conditions of the simulation of the temporal changes of the geomagnetic field, as they were defined in previous section.

\section{Spatial Modeling}

As it results from the previous section, functions $X_{k j}$ are not in obvious kind by functions of coordinates, and only by functions of a point. However, as far as points of observations are located on some surface and each point is corresponded by the definite space coordinates, the distribution $X_{k j}$ in space can be assumed not to be chaotic and described by some function of coordinates. This assumption is equal to a statement that for any point on the given area the temporal change in the geomagnetic field is a linear combination of a limited set of the temporal functions, $T_{k i}$, which are the same as on points, used for deriving NOC. Let:

$$
X_{k j}(\varphi, \lambda)=\sum_{m=0}^{M} \sum_{n=0}^{N} a_{m n} F_{m}(\varphi) \cdot G_{n}(\lambda) .
$$

Where $a_{m n}$ is numerical coefficient, and $F_{m}(\varphi)$ and $G_{n}(\lambda)$ present some analytical functions of coordinates.

It is obvious that the form of Eq. (15) is quite the same as of equations of the geomagnetic field modeling, as was described, for example, by Haines (1990). Also obvious that $X_{k j}$ is the implicit function of the field values at point $j$. Hence, all conclusions and recommendations relating to space regional modeling of the geomagnetic field, can be to the full widespread on modeling of the space distribution of $X_{k j}$. We consider some of these recommendations.

First, functions $F_{m}$ and $G_{n}$ should be chosen to satisfy conditions of a frequency filter and divide the observed field into the normal and anomalous parts on the given surface. We can hope that the completion of this condition at calculation of $X_{k j}$ will be simplified, as far as $X_{k j}$ for different $k$ are orthogonal each other. Hence, we can approve that each $X_{k j}$ has simpler distribution in space, than their sum. In other words, for its description of each $X_{k j}$ is required less of elementary functions $F_{m}$ and $G_{n}$ (less of members of exception on $n$ and on $m$ ).

Secondly, functions $F_{m}$ and $G_{n}$ should be orthogonal according to the chosen basis. For the completion of this condition, it is necessary to minimize non-diagonal terms in the matrix of the normal equation so that the solution is stabilized and becomes less dependent on restriction of $n$ and $m$. From set of possible kinds of analytical functions $F_{m}$ and $G_{n}$ we have chosen the Legandre polynomials on the $(x, y)$ plane. As it is known that these polynomials are:

$$
F_{0}=1 ; F_{1}=x ; F_{2}=\frac{\left(3 x^{2}-1\right)}{2} ; F_{3}=\frac{\left(5 x^{3}-3 x\right)}{2} ; \ldots
$$

For these functions the condition of orthogonality is fulfilled on the interval $x \in[-1 ; 1]$. So if Legandre polynomials are chosen as approximating functions in the geographical coordinates $(\varphi, \lambda)$ it is necessary to carry out their transformation to a new coordinate system $x$ and $y$ by the following formula:

$$
\begin{aligned}
x_{i} & =\frac{\varphi_{i}-1 / 2\left(\varphi_{\max }+\varphi_{\min }\right)}{1 / 2\left(\varphi_{\max }-\varphi_{\min }\right)}, \\
y_{i} & =\frac{\lambda_{i}-1 / 2\left(\lambda_{\max }-\lambda_{\min }\right)}{1 / 2\left(\lambda_{\max }-\lambda_{\min }\right)} .
\end{aligned}
$$

\section{Technique of Modeling}

As stated about determination of basic temporal functions, which are NOC in Sections 3 and 4, it is necessary to have se- 
ries of the observatories annual means being continuous during all period of modeling. Naturally such all series should belong to observatories located in an area, covered by the model. Requirements of statistics consist in that, to have much more data than parameters of model. As far as NOC's are numerical functions, each term of each NOC's should be found by means of mathematical statistics and in this sense parameters of the model should be determined. Let see the total number of NOC's. For exact description of the secular variation, $n$ is needed as the parameter number, and each temporal series contains $M$ years. So, we have $n \times M$ parameters of model. When we could use the data set at $N$ observatories, $N \times M$ of data would be available. Hence, a condition $N>n$ should be fulfilled. When $n=3$ as it happens on practice, we should have $N \gg 3$. There are not too many regions on the Earth surface where we have enough observatories which could be used for this aim. Moreover, if we have enough observatories for the temporal functions calculation, their quality does not permit arraying out the spatial modeling of the sufficient accuracy. As far as for degree of polynomials being $k$, it is necessary to have much more points then $K=(1 / 2)(k+1)(k+2)$, i.e. when $k=3$ we should have $N \gg K=10$. To overcome the first difficulty we can increase total amount of the data set with including data at observatories located in direct vicinity of the modeled area, assuming that the condition of the NOC's set for extended region is the same. To solve the second one, it is possible to use the repeat stations data inside the modeled area as additional data, although they are not the continuous temporal series.

Thus, algorithm of modeling has two stage. On the first stage, the continuous temporal series of annual means at the observatories located in an area greater than the modeled region are used. The $T_{k}(t)$ functions are determined on this stage. On second stage, the observatories located in the modeled area are again used as well as the data at repeat stations. The technique of the spatial model creation is completely the same even if the function $D(t)$ is analytical. In order to make each conditional equation for each measured value on epoch $t_{i}$, the numerical significance of $D(t)$ on the epoch is used, irrespective of deviation way of $D(t)$; i.e. it is calculated on formula or taken from table (numerical function).

Usage of the repeat station data decides one more important task. Orthogonality of the spatial functions becomes better if the distribution of repeat stations are distributed in space uniformly. From statistics it is known that to such type of distribution the summations enough fast converge to integrals; i.e. the non-diagonal elements of the matrix of normal equations become much less than diagonal ones.

The check of algorithm for modeling using test data sets has allowed to reveal two more important conditions, that are necessary to be executed at modeling. The first one is reducing errors in the initial data. What are the errors in case of the spatial modeling? First, the casual and systematic error included in observations can be thought. Secondly all anomalous field, $H_{\mathrm{a}}$, can be considered as an error for determination of the analytical function. Since $H_{\mathrm{a}}$ does not change temporally, it can be excluded. For each repeat station a conditional equation can be written as follows:

$$
\Delta H_{t_{1}-t_{2}}=\sum_{i} \sum_{j} \sum_{k} a_{i j k} F_{i}(\varphi) \cdot G_{j}(\lambda) \cdot\left[D\left(t_{1}\right)-D\left(t_{2}\right)\right] .
$$

Third type of error is close to the second one, but relates to temporal series at a observatory. If $H_{\mathrm{n}} \gg H_{\mathrm{a}}$ and $H_{\mathrm{n}}\left(t_{1}\right)-$ $H_{\mathrm{n}}\left(t_{2}\right) \ll H_{\mathrm{n}},\left(H_{\mathrm{n}}+H_{\mathrm{a}}\right)$ have to be excluded for each observatory on first stage of modeling. Practically it can be done by using as a reference epoch such one for which the best model of reference field exists. For last decades, such epoch can be 1980, because DGRF80 was designed on the MAGSAT data. It is, certainly, the best among DGRF's.

Statistical properties of a model are defined by ratio of quantities of initial data and obtained parameters of the model. It is possible in principle to describe the change of the geomagnetic field at each year with a set of annual spatial models for the temporal period, $M$. The quantity of parameters of such set of models will be $M \times K$, if the quantity of parameters of one spatial model is $K$.

As explained above, the regional orthogonal model (ROM) will have $(n \times M+n \times K)$ parameters to describe the geomagnetic field changes with the same accuracy. At $n \ll K$, as it happens on practice, quantity of ROM's parameters to be determined will be much less than those of the annual models. It means that we can determine these parameters with best accuracy, or use less amount of initial data to determine these parameters with the same accuracy. We must also emphasis that it is, in common case, impossible to create a set of equally accurate models for each year.

\section{Data for Analysis}

Three kinds of data were used. The first one is observatory network data set located within the area of modeling and around it. The list of observatories which data were used in this study is presented in Table 1 and their locations are shown in Fig. 1(a) with squares. Observatory data were used in form of 2-year interval averaged values of each geomagnetic field component referred to the beginning of the second year, i.e.

Table 1. Geomagnetic observatories used in this study and their locations.

\begin{tabular}{lcc}
\hline Observatory & Lat. $\left({ }^{\circ} \mathrm{N}\right)$ & Lon. $\left({ }^{\circ} \mathrm{E}\right)$ \\
\hline Stekolny & 60.117 & 151.017 \\
Paratunka & 53.100 & 158.633 \\
Memambetsu & 43.907 & 144.193 \\
Gornotaegnaya & 43.683 & 132.167 \\
Peking & 40.033 & 116.183 \\
Mizusawa & 39.100 & 141.200 \\
Kakioka & 36.230 & 140.190 \\
Kanozan & 35.253 & 139.960 \\
Kanoya & 31.420 & 130.882 \\
Zo-se & 31.100 & 121.183 \\
Lumping & 25.000 & 121.167 \\
\hline
\end{tabular}




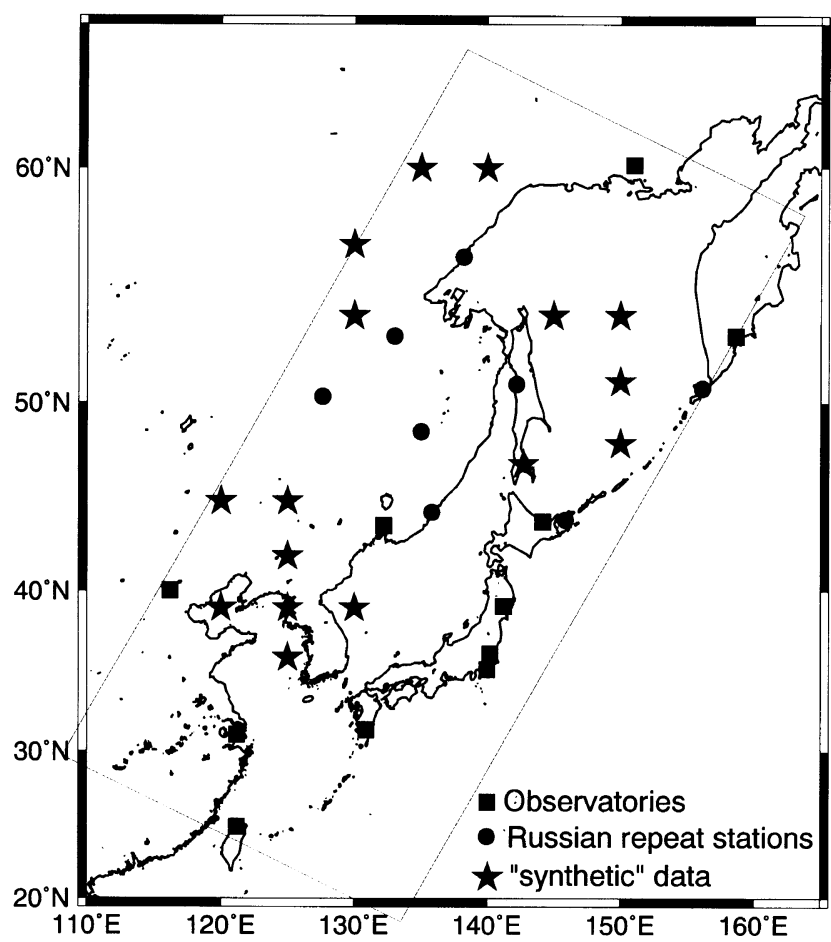

(a)

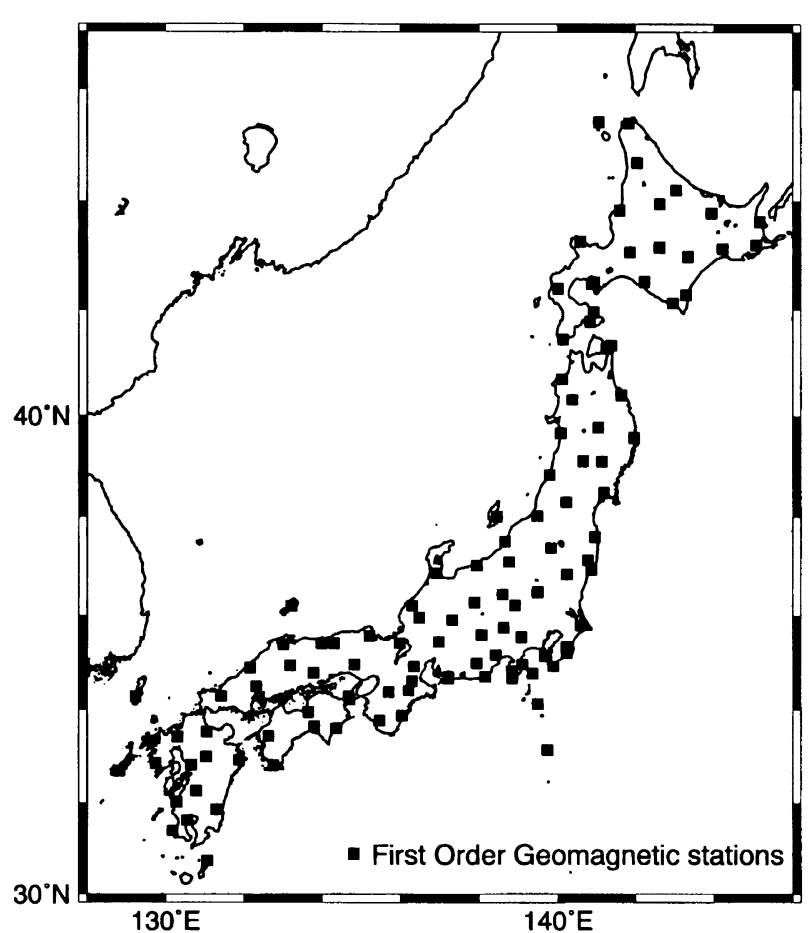

(b)

Fig. 1. (a) Data points for analysis. (b) Locations of the repeat stations (first order geomagnetic stations) in Japan used for analysis.

to $19 \mathrm{XX} .0$ epoch.

The data of the second kind being repeat station ones cover both the territories of Russia and Japan. There are 105 repeat stations of the first class in Japan and they are called the first order geomagnetic stations (Geographical Survey Institute, 1995). Observations are made every 2-5 years. Before 1987 the geomagnetic field had been measured during one quite day from 6:00 (LT) in the morning till 22:00 (LT) at night. These measurements were used for calculation of quasi-daily values which then were reduced to a year of observation 19XX.0 using the data at the Kakioka Magnetic Observatory. Since 1987 measurements have been executed with three axial fluxgate magnetometer in a full day. The averaged daily values were also reduced to a year of observation 19XX.0 using the data at Kakioka.

Data at 7 repeat stations situated in Russia were used. Observations at these stations have been made during three quite days in morning and evening hours. Every measured value was independently reduced to the annual mean of the nearest observatory. An average of all values referred to the annual mean of a year was chosen as magnetic field value at a point of observation. Further these values were also reduced to 19XX.0 epoch, where 19XX is a year of observation.

Repeat station locations are presented in Fig. 1(a) with circles, while in the Japan area the repeat stations are shown in Fig. 1(b). The square area of modeling is marked in Fig. 1(a) with straight lines.

Figure 1(a) shows that distribution of the data points within the rectangle is nonuniform. The seas are almost free of data. It is common sense that to obtain true model it is necessary to fill empty places by some set of data. So to build a spatial model of magnetic field changes, it is necessary to "fill" these "white spots". For this purpose "synthetic" data from global models IGRF (Barton et al., 1996) were used, i.e. set of 120 coefficients have been used for Spherical Harmonic Functions to get those ones. Accuracy of the IGRF system was estimated as about $50 \mathrm{nT}$ (Golovkov et al., 1997b), except DGRF80, based on large amount of magnetic components precise data from MAGSAT mission (Langel et al., 1981).

Taking into account the relatively high density of magnetic observatories in the region of modeling, we can estimate a probable inaccuracy of "synthesized" data as being less then $50 \mathrm{nT}$. Assuming random character of these errors we can use "synthesized" data from global models to fill gaps of origin data. Usage of the "synthesized" data should regularize the solution of statistical task of deriving of the ROM-Far East coefficients.

Utilizing, jointly, data of different accuracy, designers usually use different weights for these data. However, we have chosen other way of comparing RMS of the model deviations separately for each kind of data. We increased the number of "synthesized" data step by step until the RMS deviations model (ROM) data from observatory and repeat station data do not begin increase. This conditions fulfilling for this particular region leads to almost equal accuracy on the whole area under modeling, as it was obtained by a lot of tests.

The IGRF is known to be constructed as a system of spherical harmonic models for epochs being divisible by 5 and referred to the beginning of a year (19XX.0). Thus we used definitive models from the IGRF, DGRF70, DGRF75, DGRF80, DGRF85 and DGRF90, and preliminary model IGRF95. Net of additional stations was developed so that 
hollow regions on maps were uniformly covered with "synthetic" data. As a whole, there were calculated 85 values at 17 points. These points are marked with stars in Fig. 1(a). Their weight in whole amount of data was less than $20 \%$, last one is limit of amount of "synthetic" data that not disturb real data.

One can expect that total error of a model is to be about $10 \mathrm{nT}$, assuming that the error of observatory data is about $3 \mathrm{nT}$, those of the Japanese repeat stations is about $5 \mathrm{nT}$, those of Russian repeat stations is about $10 \mathrm{nT}$, and those of "synthetic" data is about $15 \mathrm{nT}$.

The most accurate model among the models composing IGRF is considered to be DGRF80 for the epoch 1980.0, which was constructed with using of large massive data of MAGSAT. In the present study, this model was used as general reference field for all kinds of data. In practice it was amounted to using numerical values of geomagnetic field element changes in respect to epoch 1980.0 as data for analysis. "Synthetic" values were obtained by calculating field increments in respect to DGRF80. As observatory data for analysis the field increments at an observatory in respect to the average of annual means of the field for epochs 1979.5 and 1980.5 were used. These two methods can not be applied to repeat station data if there were no measurements at an repeat station in 1980 . The field increments between two observations ( $\left.\Delta H=H_{19 \mathrm{XX} .0}-H_{19 Y \mathrm{Y} .0}\right)$ were used as data for analysis at the repeat stations.

For easier modeling, the transformation of coordinate system was done. Usual geographical coordinates were transformed into "reduced" coordinates. A pole of "reduced" coordinate system was chosen on the large circle drawn through the center of a line of a model in perpendicular to this line and in distance of $90^{\circ}$ from it. Thus in the new coordinate system a rectangle of a model lies around the equator and is extended along it. It is the best position for use of 2-dimensional polynomial expansion of the field, because the both coordinates have the same dimensional representation in respect to angle degrees.

In general, there were used 255 values from magnetic observatory network, 139 values from repeat stations and 85 "synthetic" values for analysis.

\section{Analysis of Temporal Functions}

To obtain a statistically substantiated expansion of temporal series in NOC's, data from 11 observatories were used (see Fig. 1(a)). They are distributed in a range of latitude from $25^{\circ} \mathrm{N}$ to $60^{\circ} \mathrm{N}$ and longitude from $116^{\circ} \mathrm{E}$ to $159^{\circ} \mathrm{E}$. From the view point of external sources, all chosen observatories concentrated in middle latitude belt do not contact directly the area of aurora and equatorial electrojet. From the view point of variation due to internal origin, they located in surrounding area of one SV focus. Temporal interval from 1970 to 1995 does not include an intensive global change in the secular variation occurred in 1969 (geomagnetic jerk).

Three enumerated conditions permit one to hope that a number of significant NOC's do not exceed the first units. As shown in previous section, the duration of a series was 26 values for epochs 19XX.0. However, since 1980.0 epoch was chosen as a basis, every row in a matrix of data consists of 25 significant digits.
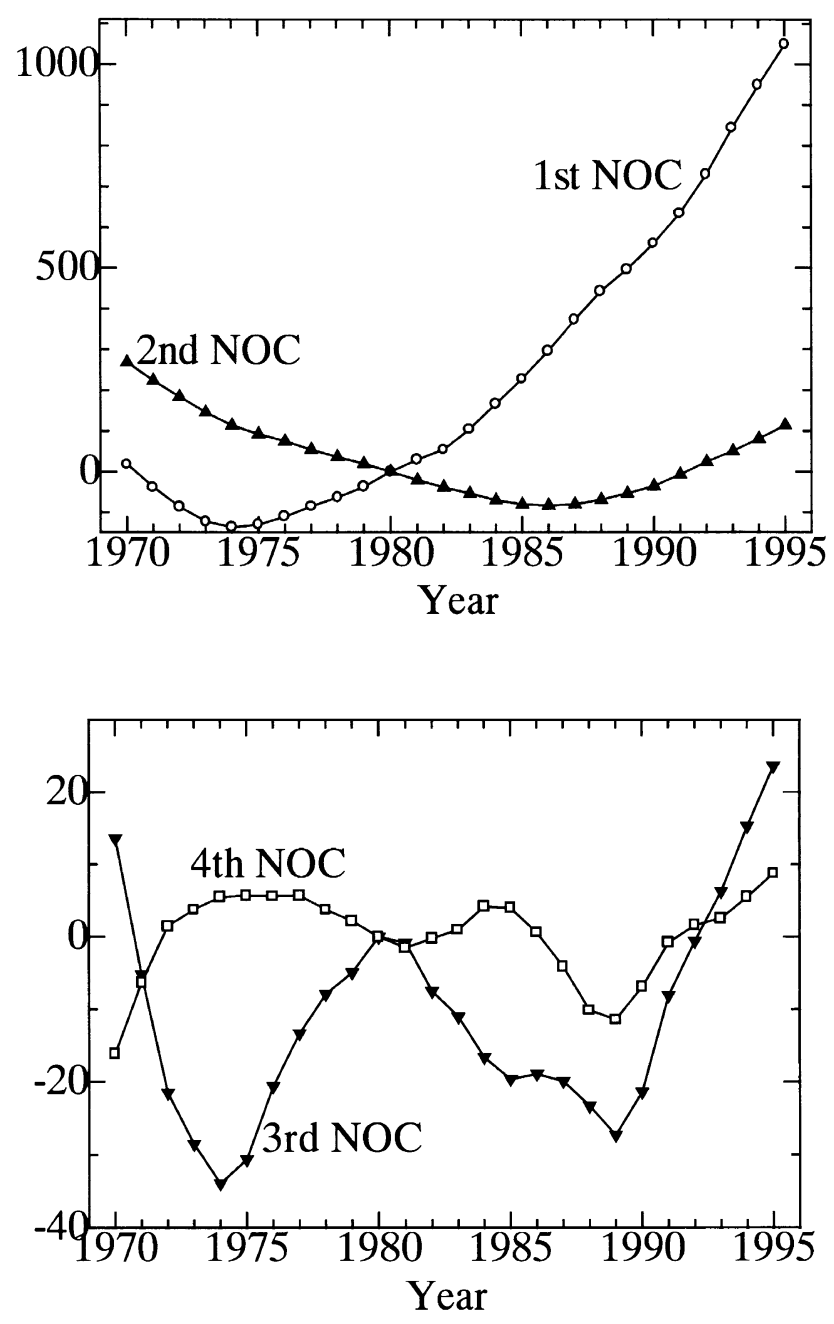

Fig. 2. Natural Orthogonal Components (NOC's) — temporal functions of the ROM-Far East model. NOC's have no unit.

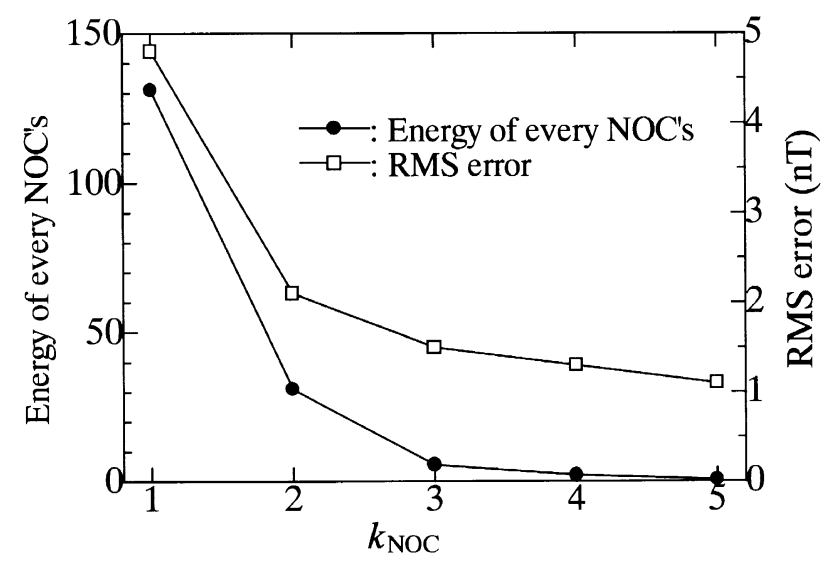

Fig. 3. Energy of every NOC's calculated as $\sigma_{\mathrm{NOC}}=$ $\sqrt{\frac{1}{286} \sum_{j}^{11} X_{k j}^{2} \sum_{i}^{26} T_{k i}^{2}}$ (no unit) and root mean square errors of the
original data approximation by the NOC-model respect to quantity of $k_{\mathrm{NOC}}$ 's. 

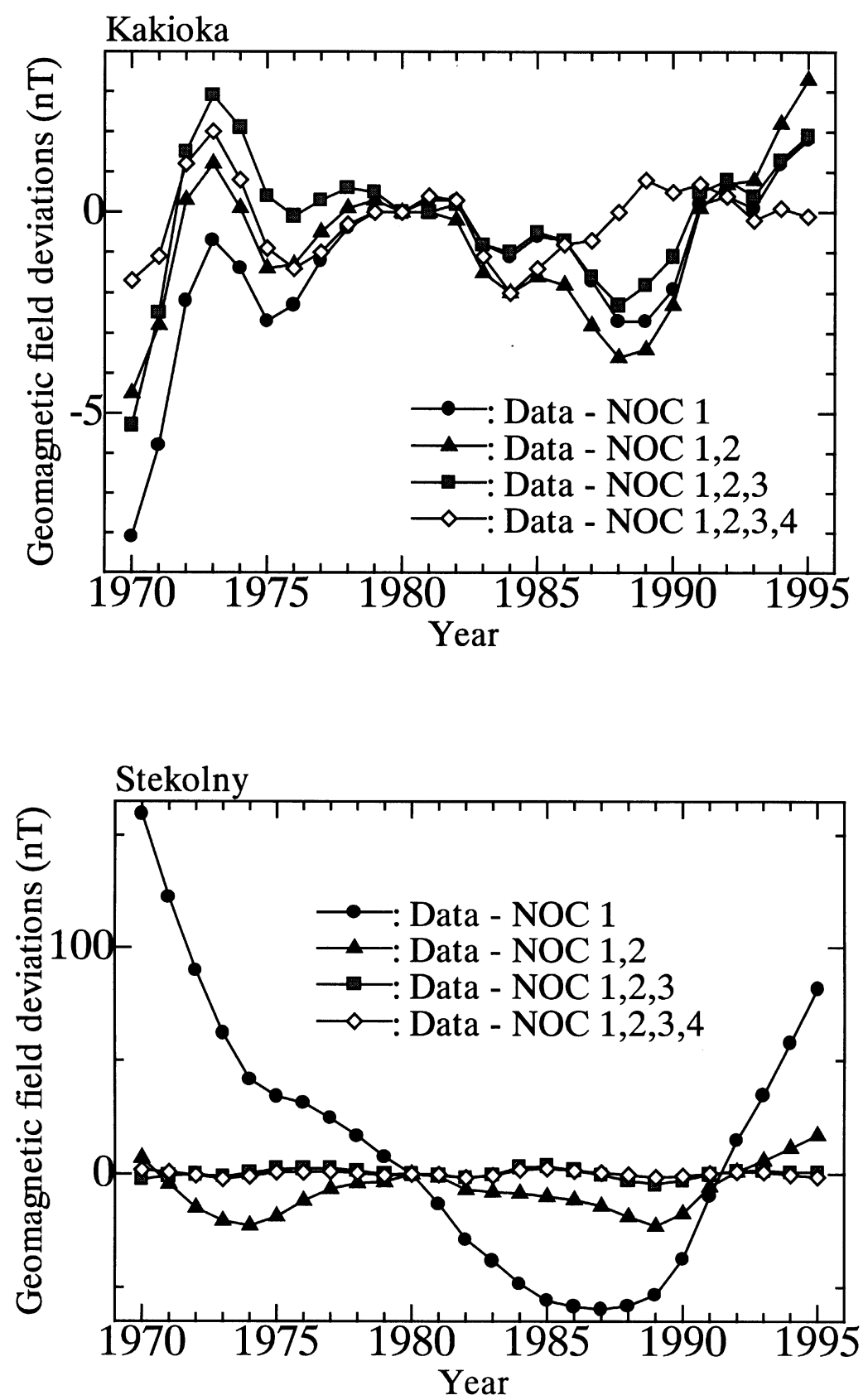

Fig. 4. Deviations between the original data and the NOC-models with the different $k_{\mathrm{NOC}}$ at the observatories of Kakioka and Stekolny.

The analysis results are presented in Figs. 2, 3 and 4. Figure 2 shows four temporal functions (NOC's). Figure 3 shows RMS input of each NOC with $k_{\mathrm{NOC}}$ (degree of NOC) calculated with the following formula:

$$
\sigma_{\mathrm{NOC}}=\sqrt{\frac{1}{286} \sum_{j}^{11} X_{k j}^{2} \sum_{i}^{26} T_{k i}^{2}}
$$

and root mean square of deviations of observed temporal series from a model with $k_{\text {NOC }}$. Figure 4 shows how model of different truncation level describe the original data series at some observatories. Root mean square errors of the modeling for all observatories and for different $k_{\mathrm{NOC}}$ are shown in Fig. 3.
Comparison of the graphs in Fig. 4 shows that residuals of the modeling for $k_{\mathrm{NOC}} \geq 3$ almost incorrelate one with another. It describes, thus, local features in each observatory record such as observatory errors and influence of any local geophysical phenomena.

As it follows from Fig. 3, the contribution of NOC with $k_{\mathrm{NOC}} \geq 4$ is small and does not influence on root mean square error of model. Figure 3 shows that residuals for $k_{\mathrm{NOC}}=3$ are within 1.5-2.0 nT, however, for some observatories they exceed $3.7 \mathrm{nT}$. Obviously, the accuracy of NOC analysis is defined by just this value and exceeding of model deviations at some observatories can be explained rather data errors at this observatory than model inaccuracy.

So, as it follows from the mentioned above, first three 


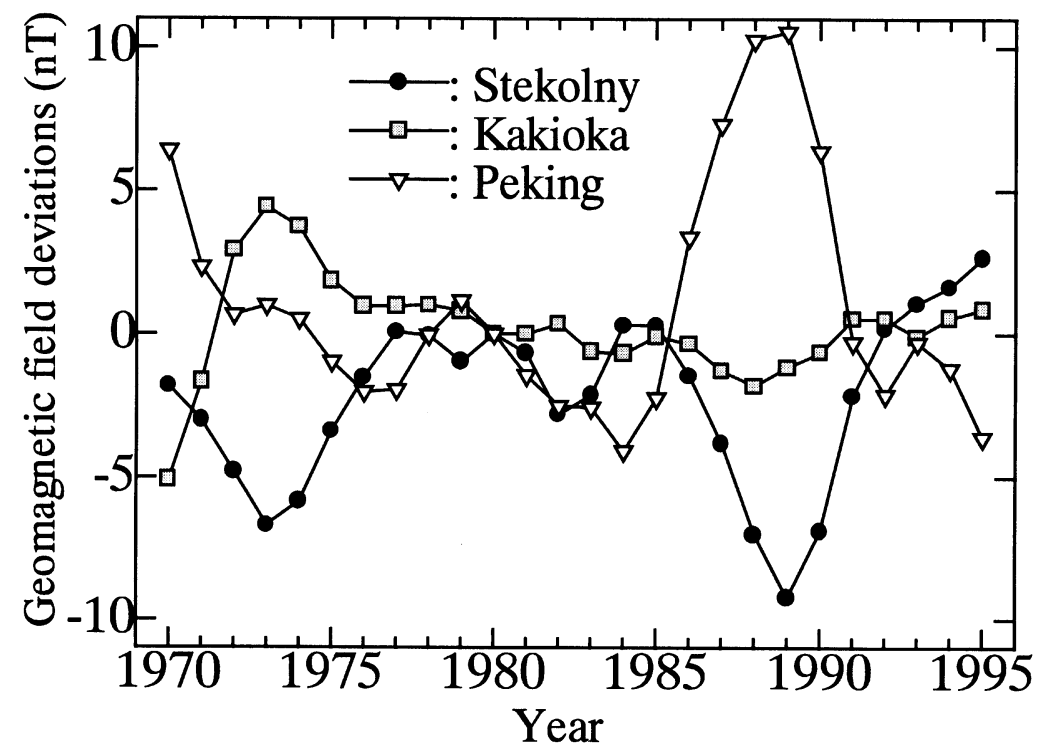

Fig. 5. Deviations between the original data and the ROM-Far East model from the observatories of Stekolny, Kakioka and Peking (secular variation of $F$ component of the geomagnetic field).

NOC's describe temporal variations of the field over this region with the accuracy of annual mean obtaining at observatories. That is why only three NOC's were chosen as basic functions for spatial-temporal analysis.

Though Fig. 4 shows that the 1969 geomagnetic jerk probably has influence on the model in the beginning of the 1970s, the influence is small and hard to distinguish it from other changes.

\section{Spatial Analysis Results}

To estimate an attainable accuracy of spatial-temporal model, built on the basis of NOC's and rectangular 2D Legendre polynomials, the following test was executed. Synthesized on grid cell whose latitudinal and longitudinal extents were $3^{\circ}$ and $5^{\circ}$ respectively, values of magnetic field of the global model, IZMST (Golovkov et al., 1997a), for 26 years were used for the rectangular model, shown in Fig. 1(a). NOC's derived from the global magnetic observatory network were used as basic temporal function of IZMST. This test became an ideal examination of the accuracy of transfer of global spherical harmonic model into polynomial one in a portion of the Earth surface of the same extent as a future model of Far East.

A model was built using polynomials of degree $n$ varying from 2 to 5. RMS differences between IZMST and regional model are presented in Table 2 . It is necessary to note that model of the fifth degree corresponds to the twelfth harmonics of the global model.

As shown in Table 2, three degree of Legendre polynomials is enough for deriving sufficient accuracy of the regional model. Just this polynomial system was chosen for designing ROM-Far East. Total number of coefficients was $3 \times 10=30$. A system of conditional equations included 479 equations while using data described in Section 6, i.e. it was overdeterminated by 16 times.

RMS errors of initial data description appeared to be $4.9 \mathrm{nT}$
Table 2. RMS differences between IZMST and regional model.

\begin{tabular}{ccccc}
\hline$n$ & 2 & 3 & 4 & 5 \\
\hline$\sigma(\mathrm{nT})$ & 2.1 & 1.5 & 1.3 & 1.1 \\
\hline
\end{tabular}

$n$ : polynomial degree.

for observatories, $6.9 \mathrm{nT}$ for repeat stations and $26.7 \mathrm{nT}$ for values synthesized by using global model coefficients. Commonly for all types of data RMS errors were $12.4 \mathrm{nT}$.

So large errors can be explained by the fact that values synthesized with IGRF are significantly different from measured data for this region. Table 3 shows the deviations of IGRF from the magnetic field values at observatories situated in this region and reduced to 1980.0 epoch (DGRF80 is considered to have a zero error). As one can see from Table 3 deviations at some observatories reach $80 \mathrm{nT}$.

To correct the situation global models IGRF were "improved" by use of data at the observatories situated in this region. For this purpose every column of data from Table 3 was used for derivation of additional polynomial model of the first degree covering the area of modeling. These models describe the spatial distribution of additions to global model for each epoch being divisible by 5 years.

By using the experimental data from observatories and repeat stations as well as improved (corrected) synthetic data a finite model ROM-Far East was designed. RMS differences between model and observatory data are $3.8 \mathrm{nT}$, those for repeat stations data are $6.3 \mathrm{nT}$, those for synthetic data are $9.3 \mathrm{nT}$, and as a whole $5.9 \mathrm{nT}$ (Table 4 ). The graph of temporal dependence of initial data and the same of deviations of this data from the model are shown in Fig. 5. The variation of Kakioka is rather smoother than that of Stekolny and Peking because there are more data, such as repeat stations and other observatories, around Kakioka. 
Table 3. Deviations of IGRF from the magnetic field values (nT) from observatories situated in this region and reduced to 1980.0 epoch (DGRF80 is considered to have a zero error).

\begin{tabular}{lrrrrr}
\hline Observatory & 1970 & 1975 & 1985 & 1990 & 1995 \\
\hline Stekolny & 6.8 & 11.3 & -8.3 & -14.6 & 17.4 \\
Paratunka & 24.7 & 55.0 & 3.0 & 16.0 & 41.8 \\
Memambetsu & -34.7 & 19.1 & 16.1 & 29.3 & 53.9 \\
Gornotaegnaya & -28.3 & 20.5 & 12.6 & 35.6 & 71.2 \\
Peking & -1.0 & 37.9 & -7.2 & 13.0 & 63.4 \\
Mizusawa & -39.1 & 11.0 & 15.2 & 24.8 & 53.1 \\
Kakioka & -37.2 & 8.6 & 13.7 & 18.4 & 49.4 \\
Kanozan & -37.3 & 7.2 & 13.4 & 16.9 & 47.7 \\
Kanoya & -21.5 & 10.1 & 9.6 & 6.1 & 40.1 \\
Zo-se & 10.1 & 44.6 & 38.6 & 38.7 & 81.3 \\
Lumping & 16.9 & 16.3 & 21.6 & 5.6 & 31.0 \\
\hline
\end{tabular}

Table 4. Number of data and RMS differences (nT) between model and observatory data.

\begin{tabular}{lcccc}
\hline & Observatory & Repeat stations & IGRF & Total \\
\hline ROM "FAR EAST" & $225 / 3.8$ & $169 / 6.3$ & $85 / 9.3$ & $479 / 5.9$ \\
ROM "JAPAN" & $125 / 2.0$ & $740 / 5.4$ & - & $865 / 5.0$ \\
\hline
\end{tabular}

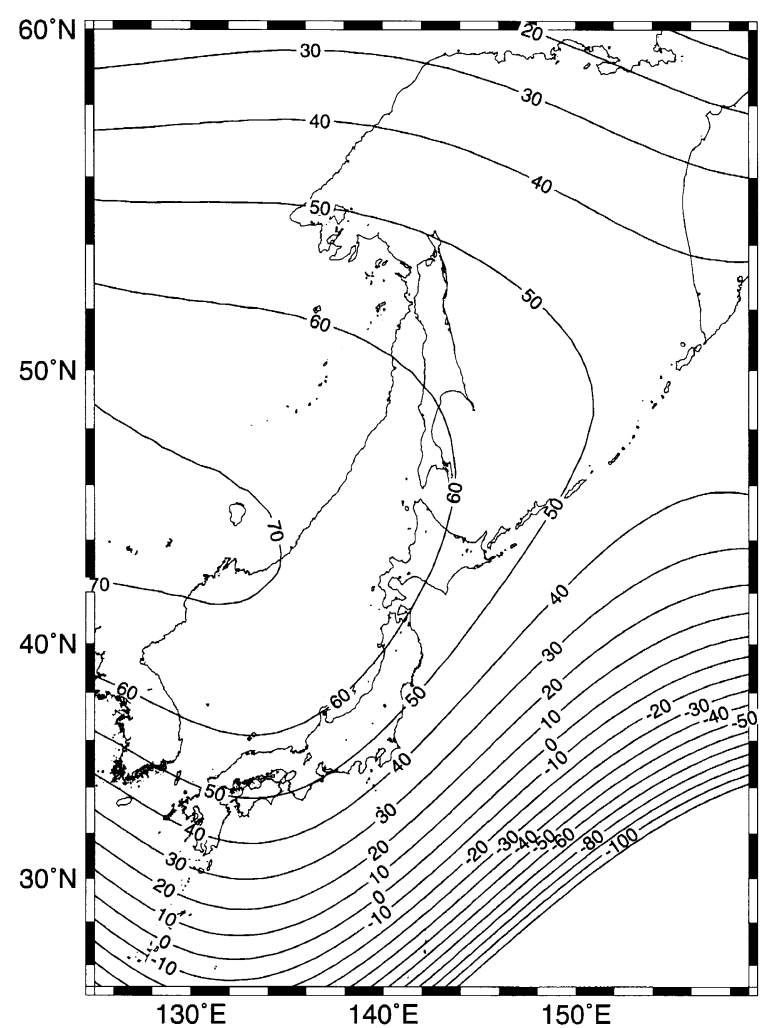

Fig. 6. Differences of the changes of the geomagnetic field during 1980-1995 calculated with ROM and IGRF95. The unit for contours is given in $\mathrm{nT}$.
Polynomial modeling to third degree was executed for comparison over the smaller area covered only Japanese islands, using experimental data from 5 magnetic observatories and from 144 repeat stations. In general 865 conditional equations were formed. Errors of the model were $2.0 \mathrm{nT}$ for observatories, $5.4 \mathrm{nT}$ for repeat stations and $5.0 \mathrm{nT}$ as a whole (Table 4, last line).

A comparison of the regional model and IGRF was made for the 1995 epoch. Changes of the geomagnetic field on the time interval 1980-1995 were calculated with ROM and IGRF95. Differences of the modeled fields are presented in Fig. 6. As seen in the figure, they reach $60 \mathrm{nT}$ in the area of the regional model. Taking into account a rather good agreement between these observatory time series and the regional model (see Fig. 5), disagreement between the ROM and IGRF shows the largest discrepancy of the global model in this area.

\section{Conclusions}

Comparing the accuracy of global and regional models, one can conclude that the accuracy of regional model is higher significantly. For the areas covered by the high dense network of observatories and repeat stations, this conclusion is considered to be real. However, it is possible to reach high accuracy of regional modeling even for Far East, where seas form the sufficient part of the area. For this purpose it is necessary to add the data synthesized by use of global model coefficients. However, the main assumption is that the secular variation for present time interval at any point 
of modeling area is a linear combination of limited number of independent variations. Thus the problem of modeling can be reduced to spatial description of coefficients in linear combination pointed out previously. ROM-Far East, being described in this study, is based on modeling of spatial distribution of three variations.

Unfortunately, it was revealed during this study that the errors of widely accepted global model IGRF for this region appeared to be significant. The designing of the regional model using only experimental data from this region appeared to be impossible because of rather nonuniformity of data spatial distribution. Nevertheless these data permitted improving the global model. The used technique of improving is equivalent to an assumption that the global model for each epoch is sufficiently describe "a curvature" of the geomagnetic field distribution. Hence the high accuracy of the regional model is provided by two rather reliable assumptions considering temporal and spatial variability of the field and about rather great amount and high accuracy of experimental data concentrated in this area.

Acknowledgments. We thank Y. Nishida and N. Oshiman for their careful reviews and constructive comments.

\section{References}

Barton, C. E., R. T. Baldwin, D. R. Barraclough, S. Bushati, M. Chiappini,
Y. Cohen, R. Coleman, G. Hulot, P. Kotzé, V. P. Golovkov, A. Jackson, R. A. Langel, F. J. Lowes, D. J. McKnight, S. Macmillan, L. R. Newitt, N. W. Peddie, J. M. Quinn, and T. J. Sabaka, International Geomagnetic Reference Field. 1995 Revision. IAGA Division V. Working Group 8, Phys. Earth Planet. Inter., 97, 23-26, 1996.

Fainberg, E. B., The division geomagnetic of fields on normal and abnormal part, Geomagn. Aeron., 15, 144-148, 1975.

Geographical Survey Institute, First order geomagnetic survey in Japan from 1949 to 1994, Technical Rep. Geograph. Surv. Inst., B4-12, 174 pp., 1995.

Golovkov, V. P., G. Kozhoeva, and S. I. Shkolnikova, The use of method natural orthogonal component for division partially non-orthogonality variations geomagnetic of field, Geomagn. Aeron., 32, 162-165, 1992.

Golovkov, V. P., T. N. Bondar, I. A. Burdelnaya, and S. V. Yakovleva, Using satellite magnetic surveys data for the spatial-temporal modeling of the geomagnetic secular variation, J. Geomag. Geoelectr., 49, 207-227, $1997 \mathrm{a}$.

Golovkov, V. P., T. N. Bondar, I. A. Burdelnaya, and S. V. Yakovleva, Comparison of candidate models for DGRF 1990 and IGRF 1995, J. Geomag. Geoelectr., 49, 291-315, 1997b.

Haines, G. V., Regional magnetic field modeling: A. Review, J. Geomag. Geoelectr., 42, 1001-1018, 1990.

Langel, R. A., J. T. Robert, and R. Horner, Magsat data processing: a report for investigator, NASA/GSFC Technical Memorandum, 82160, 178 pp., 1981.

Vertlib, À. B. and X. Y. Vagner, The analysis geomagnetic Sq-variations with help of method of decomposition of fields on natural orthogonal component, Geomagn. Aeron., 10, 649-654, 1970.

I. A. Burdelnaya, S. V. Filippov (e-mail: sfilip@izmiran.troitsk.ru), V. P. Golovkov (e-mail: golovkov@izmiran.troitsk.ru), S. Fujiwara (e-mail: fujiwara@gsi-mc.go.jp), T. Tanabe, S. Nishi, M. Kaidzu, and S. Matsuzaka 